\title{
Dormancy Induces the Formation of Persisters in the Candida Albicans-staphylococcus Epidermidis Mixed Species Biofilm
}

jichen Yang

960th Hospital of PLA

Bingquan Xiang

Yunnan Cancer Center: Yunnan Cancer Hospital

\section{Wei Wang}

Yunnan Cancer Hospital

\section{Sunyin Rao}

Yunnan Cancer Hospital

\section{Run Cao}

Yunnan Cancer Hospital

Shouyong Xiao

Yunnan Cancer Hospital

\section{Xin Cui}

Yunnan Cancer Hospital

\section{Yunchao Huang}

Yunnan Cancer Center: Yunnan Cancer Hospital

lianhua ye ( $\sim$ yelianhuaa@163.com )

Yunnan Cancer Center: Yunnan Cancer Hospital https://orcid.org/0000-0002-7748-5830

\section{Research article}

Keywords: Candida albicans, Staphylococcus epidermidis, mixed biofilm, persisters, dormancy induction

Posted Date: May 6th, 2021

DOl: https://doi.org/10.21203/rs.3.rs-493339/v1

License: (1) (i) This work is licensed under a Creative Commons Attribution 4.0 International License.

Read Full License 


\section{Abstract}

Background: Persisters make biofilm infections difficult to cure, the low content of persisters in biofilm and the difficulty of obtaining them are outstanding problems in research, finding a reliable method to obtain a sufficient amount of persisters is the key to research.

Methods: In this study, C.albicans strain ATCC10231 and S.epidermidis ATCC35984 were used to culture mixed biofilms on the surface of a titanium metal coupon. Tetracycline, gentamicin, 5-fluorocytosine(5Fc), caspofungin, rifampicin, fluconazole and carbonyl cyanide m-chlorophenylhydrazone (CCCP) were used to induce the dormancy of biofilms to produce persisters. Ciprofloxacin and amphotericin B were used to kill the non dormant bacteria, and then the persisters were screened.

Results:The study showed that dormancy induced by tetracycline and 5-Fc could significantly increase the persister levels of S.epidermidis and C.albicans, respectively. After the induced persisters were recultured, there was no significant change in antibiotic sensitivity compared with before induction.Scanning electron microscope(SEM) observations showed that after dormancy induction, the surface morphology of C.albicans in the mixed biofilm showed convex changes, and the volume of S.epidermidis decreased. Confocal laser scanning microscope (CLSM)observation showed that the mixed biofilm with high content of persisters did not dissolve under the killing of antibiotics, and the biofilm with low content of persisters dissolved in large area.

Conclusions: Induction of dormancy is a reliable method for the formation of persistent bacteria in C.albicans-S.epidermidis mixed biofilm.

\section{Background}

The development of medicine makes more and more medical devices used to treat human disease, the incidence of biofilm infection in these patients with indwelling medical devices is also increasing[1], which can cause the dysfunction of the device that is implanted[2] and serious complications[3]. For example, the incidence of infective endocarditis after transcatheter aortic valve implantation was $5.5 \%$, after the occurrence of infective endocarditis, the in-hospital mortality was $36.4 \%$, and the one-year mortality rate was as high as 54.5\%[4]. S.epidermidis has now become one of the most prevalent causes for implant-associated and nosocomial infections[5], such as endotracheal intubation devices[6] and artificial heart valves[7]. Candida is a kind of opportunistic pathogen, which causes systemic infection through the colonization of oral, gastrointestinal and urinary tract[8]. C.albicans can form biofilms on almost any medical device, including urinary catheters, prosthetic joints, cardiac valves, pacemakers[9]. Of all types of nosocomial infections, approximately $27 \%$ of nosocomial candida infections are polymicrobial[10]. During long-term infection, multiple pathogenic microorganisms will produce mixed infections, and form mixed biofilm on the surface of biomaterials, the pathogenic bacteria will have a synergistic effect to resist the killing of antibiotics, making the infections difficult to eradicate[11]. 
Persister cells are a slow-growing semi-dormant fraction of cells within a biofilm with low levels of transcriptional activity[12], the reductions of anabolism and energy metabolism are their important characteristics[13]. The induced dormancy that characterizes the persistent cell population makes them resistant to classical antibiotics, and is one of the main reasons for biofilm recalcitrance[14]. But persisters comprise less than $1 \%$ of cells in a biofilm, one of the key challenges in the study is the difficulties with isolation maintenance of the persistent cell phenotype during culture[15]. However, there have been no reports of large number of induced persisters of S.epidermidis and C.albicans. Study found that inhibiting cellular transcription, translation, or the formation of adenosine triphosphate increases persister cell formation inEscherichia coli[16], aminoglycoside antibiotics combined with bacterial metabolites could reduce the formation of persisters and increase the killing capacity of antibiotics[17]. Based on these studies, we speculate, the use of specific drugs to block the energy metabolism of bacteria and fungi can induce cells to enter a dormant state, it is possible to produce a large number of persistent cells.

This study builds upon on our previously established polymicrobial biofilm model, in which a mixture of S.epidermidis and C.albicans were co-cultured on a biomaterial (e.g. PVC, titanium)[18], to determine the level of persisters in the mixed biofilms. The structural relationship between persisters and the biofilm was observed using SEM and CLSM. Our research solved the technical dilemma in studying the rare phenotypes of microorganisms, and contribute to understand the formation of the persister phenotype in polymicrobial biofilms.

\section{Methods}

\subsection{Bacterial and fungal strains}

Standard S.epidermidis strain ATCC35984 and C.albicans strain ATCC10231 used in this study were purchased from the American Type Culture Collection(ATCC). Live/Dead BaclightTM Bacterial Viability Kit7017 was purchased from Invitrogen, USA.

\subsection{Establishment of a S.epidermidis-C.albicans polymicrobial mexed biofilm model}

S.epidermidis strain and C.albicans strain was sub-cultured on Sabouraud agar plate(Beijing Solaibao Technology Co., Ltd. China) and Mueller Hinton (MH) agar plate(Beijing Solaibao Technology Co., Ltd. China) overnight at $37^{\circ} \mathrm{C}$, respectively. Isolated colonies were picked and inoculated into TBS and grown at $37^{\circ} \mathrm{C}$ in a $150-r p m$ shaker for $24 \mathrm{~h}$ until the logarithmic growth phase. The optical density (OD) of C.albicans suspension and S.epidermidis suspension were used to adjust cultures to a final concentration of $2 \times 10^{6} \mathrm{CFU} / \mathrm{mL}$ by adding sterilized TSB medium. The bacterial and the fungal suspensions were then mixed in 1:1 volume ratio to obtain a polymicrobial mixture containing each microbe at concentration of $1 \times 10^{6} \mathrm{CFU} / \mathrm{mL}$. Ten milliliters of the polymicrobial mixture was used for bacterial identification and drug 
sensitivity detection in a fully automated system. The polymicrobial mixture was divided into 9 test tubes, each containing a sterilized $1 \mathrm{~cm} \times 1 \mathrm{~cm} \times 1 \mathrm{~mm}$ titanium coupon (sterilized at $121^{\circ} \mathrm{C}$ for $30 \mathrm{~min}$ ), and incubated at $37^{\circ} \mathrm{C}$ in a 150 -rpm shaker for $24 \mathrm{~h}$ to allow C.albicans and S.epidermidis to adhere to the surface of the coupon.

\subsection{Dormancy induction}

Titanium coupons with adhered cells were transferred to the fresh TSB containing tetracycline, gentamicin, rifampicin, 5-FC, caspofungin, fluconazole, and CCCP(Beijing Solaibao Technology Co., Ltd. China) solutions(all at $10,000 \mu \mathrm{g} / \mathrm{mL}, 100 \mu \mathrm{L}$ )for dormancy induction, and TSB without antibiotics was used as control. By inhibiting the anabolism of microorganisms, C.albicans and S.epidermidis in the mixed biofilm were induced to enter dormant state. After incubating in a 30 -rpm shaker at $37^{\circ} \mathrm{C}$ for $1 \mathrm{~h}$, the media was removed and replaced with TSB containing $10 \mu \mathrm{g} / \mathrm{mL}$ ciprofloxacin and amphotericin B in the experimental groups and the negative control group, or sterilized TSB in the blank control group, all the above antibiotics were 10 times minimal inhibitory concentration(MIC). Cells were subsequently incubated in a $30-\mathrm{rpm}$ shaker at $37^{\circ} \mathrm{C}$ for $6 \mathrm{~h}$. By inducing dormancy treatment, non persistent bacteria cells will be killed by high concentration of antibiotics, and only persistent bacteria cells can survive. The 9 titanium coupons were then transferred to a new centrifuge tube containing $10 \mathrm{~mL}$ sterilized TSB medium, and were sonicated to dislodge the polymicrobial biofilms into the aqueous phase. Ten microliters of this polymicrobial solution was spread on an $\mathrm{MH}$ agar plate and a Sabouraud agar plate, and incubated at $37^{\circ} \mathrm{C}$ for $24 \mathrm{~h}$ for enumeration. C.albicans cannot grow on $\mathrm{MH}$ agar plate, and S.epidermidis can not grow on Sabouraud agar plate. By this method, the number of S.epidermidis and C.albicans living on the surface of each biomaterial could be counted respectively. The count of the blank control group shows the number of S.epidermidis and C.albicans on the surface of the biological material after $24 \mathrm{~h}$ of normal culture. The count of the negative control group showed the level of naturally occurring persister cells in the C.albicans-S.epidermidis mixed biofilm. The counts of each experimental group represent the proportion of persistent cells produced in the mixed biofilm after induction treatment with each antimetabolites. The above experiment was repeated 15 times. Based on initial results, tetracycline, gentamicin, 5-FC, caspofungin were selected for subsequent experiments.

\subsection{Drug sensitivity tests}

The microbial colonies in the agar plate before sterilization and after dormant sterilization were used to prepare microbial suspensions, which were subjected to microbial identification/drug sensitivity in an automated system to exclude the possibility of antimicrobial drug-resistance(Table 2).

\subsection{Sample preparation and observation with SEM}


After the induction of dormancy, titanium coupons from the 4 experimental groups and the blank control group were placed in a 24-well plate, and washed 3 times in $1 \mathrm{~mL}$ cold PBS. Biofilms were fixed in $2.5 \%$ glutaraldehyde solution for $24 \mathrm{~h}$. After washing 3 times in PBS, biofilms were fixed in $1 \%$ osmium acid solution at $4^{\circ} \mathrm{C}$ for $2 \mathrm{~h}$, followed by dehydration in gradient ethanol solution for $20 \mathrm{~min}$, isoamyl acetate replacement for $20 \mathrm{~min}$, tert-butanol penetration at $40^{\circ} \mathrm{C}$ for $2 \mathrm{~h}, \mathrm{CO}_{2}$ critical drying, ion-sputtered surface fixed coating, and SEM(Laica, Germany) observation of the ultrastructure of the biofilms.

\subsection{Sample preparation and observation with CLSM}

Biofilms on titanium coupons prepared as described above were divided into three groups: blank control group (non-dormancy-induced and non-sterilized), negative control group (non-dormancy-induced, but sterilized), and experimental group (dormancy-induced and sterilized). Fluorescent dyes for bacterial viability assay were prepared using Live/Dead Baclight TM Bacterial Viability Kit 7017(Invitrogen, USA) following manufacturer's instructions. Coupons were washed 4 times in $4 \mathrm{~mL} 4^{\circ} \mathrm{C}$ cold PBS and stained using $400 \mu \mathrm{L}$ Live/Dead microbial staining reagents in the dark at room temperature for $20 \mathrm{~min}$ according to the manufacturer's instructions. The biofilm ultrastructure of samples were observed by CLSM(CLSMFV1000, Olympus Corporation, Japan) equipped with an argon laser (514/488 nm). The number of bacterial colonies in a unit of field of view was counted by randomly examining one field of view for samples of each group. The thickness of biofilm was measured by collecting confocal images, with resolution of 1024 pixels, in serial sections at $1 \mu \mathrm{m}$ intervals from interior toward exterior of one bacterial colony randomly selected from each field of view.

\subsection{Statistical analysis}

SPSS23.0 statistical software (IBM SPSS Inc., Chicago, IL) were used to compare the counts of each experimental group with the counts of the negative control group and the blank control group. The comparison between groups was analyzed by independent sample $t$-test; $P<0.05$ was considered statistically significant.

\section{Results}

\section{1. dormancy induction increased the formation of persisters in the mixed biofilm}

Compared with the negative control group, the dormancy induced by tetracycline, gentamicin, rifampicin, $5-\mathrm{FC}$, fluconazole and CCCP significantly increased the level of S.epidermidis persisters $(P<0.01)$, and the level of persisters of the tetracycline group was the highest $(P<0.01)$. The dormancy induced by $5-\mathrm{Fc}$, and caspofungin significantly increased the level of $C$.albicans persisters $(P<0.01)$, and the level of persisters of the 5-Fc group was the highest $(P<0.01)$ (Table 1). The bacterial solution of mixed biofilm in blank 
control group (not induced dormancy, not sterilized), negative control group (after induced dormancy, but not sterilized), and the mixed biofilm bacterial solution obtained by reculture in the experimental group(after dormancy induction, and after sterilization), the results of strain identification were $S$. epidermidis and C.albicans. There was no contamination of bacteria in the operation process. According to the drug sensitivity test, the S.epidermidis strain before inducing dormancy was resistant to erythromycin, clindamycin, oxacillin, and penicillin; and was sensitive to ciprofloxacin, tetracycline, gentamicin, rifampin, linezolid, moxifloxacin, nitrofurantoin, levofloxacin, tigecycline, and vancomycin.

Table 1

The colony count of S.epidermidis and C.albicans in each group after dormancy induction $( \pm \mathrm{s})$

\begin{tabular}{|c|c|c|c|c|}
\hline group & $\begin{array}{l}\text { Dormancy } \\
\text { inducing drugs }\end{array}$ & $\begin{array}{l}\text { Drugs to kill non persister bacteria } \\
\text { (ciprofloxacin }+ \text { amphotericin } B \text { ) }\end{array}$ & $\begin{array}{l}\text { S.epidermidis } \\
\left(\times 10^{4} \mathrm{CFU}\right)\end{array}$ & $\begin{array}{l}\text { C.albicans } \\
\left(\times 10^{4}\right. \\
\text { CFU) }\end{array}$ \\
\hline 1 & tetracycline & yes & $46.17 \pm 0.85^{\star}$ & $\begin{array}{l}0.39 \pm \\
0.052\end{array}$ \\
\hline 2 & gentamicin & yes & $39.27 \pm 1.10$ & $\begin{array}{l}0.67 \pm \\
0.073\end{array}$ \\
\hline 3 & rifampicin & yes & $1.87 \pm 0.20$ & $\begin{array}{l}0.51 \pm \\
0.062\end{array}$ \\
\hline 4 & $5-\mathrm{Fc}$ & yes & $1.51 \pm 0.22$ & $\begin{array}{l}21.96 \pm \\
0.86^{\star \star}\end{array}$ \\
\hline 5 & caspofungin & yes & $1.14 \pm 0.23$ & $\begin{array}{l}14.75 \pm \\
0.65\end{array}$ \\
\hline 6 & fluconazole & yes & $2.41 \pm 0.15$ & $\begin{array}{l}8.41 \pm \\
0.59\end{array}$ \\
\hline 7 & $\mathrm{CCCP}$ & yes & $2.23 \pm 0.43$ & $\begin{array}{l}0.53 \pm \\
0.078\end{array}$ \\
\hline $\begin{array}{l}\text { 8(negative } \\
\text { control) }\end{array}$ & none & yes & $1.03 \pm 0.21$ & $\begin{array}{l}0.73 \pm \\
0.077\end{array}$ \\
\hline $\begin{array}{l}9 \text { (Blank } \\
\text { control) }\end{array}$ & none & none & $73.26 \pm 1.02$ & $\begin{array}{l}42.16 \pm \\
0.89\end{array}$ \\
\hline \multicolumn{5}{|c|}{$\begin{array}{l}\text { *Compared with the negative control group, the dormancy induced by tetracycline, gentamicin, } \\
\text { rifampicin, } 5 \text {-FC, fluconazole and CCCP significantly increased the level of } S \text {. epidermidis persisters }(P \\
<0.01) \text {, and the level of persisters of the tetracycline group was the highest }(P<0.001) \text {. }\end{array}$} \\
\hline \multicolumn{5}{|c|}{$\begin{array}{l}\star \star \text { Compared with the negative control group, the dormancy induced by } 5-\mathrm{Fc} \text {, caspofungin, } \\
\text { andcaspofunginsignificantly increased the level of } C \text {.albicans persisters }(P<0.01) \text {, and the level of } \\
\text { persisters of the } 5 \text {-Fc group was the highest }(P<0.001) \text {. }\end{array}$} \\
\hline
\end{tabular}

The C.Albicans strain was sensitive to amphotericin $\mathrm{B}$, fluconazole, 5-FC, caspofungin, and itraconazole. Induction of dormancy did not result in resistance of S.epidermidis and C.albicans to the antimicrobials 
tested. After treatment with ciprofloxacin and amphotericin B, the survival of some S.epidermidis and C.albicans was not caused by antibiotic resistance due to drug-resistant mutation (Table 2).

\section{Table 2}

The agents susceptibility to S.epidermidis and C.albicans before and after dormancy induction

\begin{tabular}{|c|c|c|}
\hline Antimicrobial drug & MICbefore induction $(\mu \mathrm{g} / \mathrm{mL})$ & MICafter induction $(\mu \mathrm{g} / \mathrm{mL})$ \\
\hline \multicolumn{3}{|l|}{ S. epidermidis } \\
\hline Ciprofloxacin & $\leq 0.5$ & $\leq 1$ \\
\hline Tetracycline & $\leq 1$ & $\leq 2$ \\
\hline Gentamicin & $\leq 4$ & $\leq 4$ \\
\hline Rifampin & $\leq 0.5$ & $\leq 1$ \\
\hline Linezolid & $\leq 2$ & $\leq 2$ \\
\hline Moxifloxacin & $\leq 0.25$ & $\leq 0.25$ \\
\hline Nitrofurantoin & $\leq 16$ & $\leq 16$ \\
\hline Levofloxacin & $\leq 0.125$ & $\leq 0.125$ \\
\hline Tigecycline & $\leq 0.125$ & $\leq 0.125$ \\
\hline Vancomycin & $\leq 2$ & $\leq 2$ \\
\hline Erythromycin & $>8$ & $>8$ \\
\hline Clindamycin & $>8$ & $>8$ \\
\hline Oxacillin & $>4$ & $>4$ \\
\hline Penicillin & $>0.5$ & $>0.5$ \\
\hline \multicolumn{3}{|l|}{ C.albicans } \\
\hline Amphotericin B & $\leq 0.5$ & $\leq 1$ \\
\hline Fluconazole & $\leq 1$ & $\leq 2$ \\
\hline $5-\mathrm{FC}$ & $\leq 4$ & $\leq 4$ \\
\hline Caspofungin & $\leq 0.125$ & $\leq 0.125$ \\
\hline Itraconazole & $\leq 0.125$ & $\leq 0.125$ \\
\hline
\end{tabular}




\subsection{Morphological and structural changes of persisters in the mixed biofilm under SEM}

Under SEM, the blank control group, the negative control group, and the experimental group all showed that mixed growth of C.albicans and S.epidermidis in the mixed biofilms. The hyphae and spores of C.albicans formed the reticular structure skeleton of the mixed biofilm. In the skeleton, S.epidermidis adheres to the surface of C.albicans, gathers together to form a mass, dense and complex hybrid biofilm. The morphological characteristics are consistent with the previous research results of the research group[18]. In the experimental group, the number of mixed biofilm reduced, the density of structure decreased, the clumpy colonies decreased, and the number of bacteria and fungi is reduced(Fig. $1 \mathrm{~A}, \mathrm{~B})$.

The mixed biofilm treated with 5-FC and caspofungin to induce dormancy, although the ratio of yeast phase to mycelium phase of $C$.albicans did not change, but the surface of $C$.albicans has a convex morphological change, with a diameter of about $70 \%-80 \%$ of the host bacteria(Fig. $1 \mathrm{C}, \mathrm{D}$ ), the change was noted to occur in both the yeast and the mycelial phases. In the negative control group, the C.albicans in the mixed biofilm had almost no such morphological changes(Fig. 1E). Afterinduced by tetracycline and gentamicinin the experimental group, the S.epidermidis showed reduced cell division and smaller volume than normal cells(Fig. 1F, G). In the mixed biofilm in the blank control group, there were a large number of mitotic cells with uniform size (Fig. 1H).

\subsection{Changes of persisters and mixed biofilms under CLSM}

In the blank control group, the CSLM images showed that mixed biofilm adhered to the surface of biomaterials, the bacteria on the surface of the biofilm are mainly living, which emit green fluorescence, and are densely distributed in clumps. The average thickness of biofilm is about $37.1 \mu \mathrm{m}$ (Fig. $2 \mathrm{~A}$ ). In the negative control group, the mixed biofilm was dissolved and destroyed, and a large number of dead bacteria were seen, which emit red fluorescence. The live bacteria were distributed on the surface of the mixed biofilm in a dot shape, and hardly gather in clumps, the average thickness of biofilm is about $6.3 \mu \mathrm{m}$ (Fig. 2B). In the experimental group, the structure of the mixed biofilm was partially destroyed, the average thickness is about $15.7 \mu \mathrm{m}$. The mixed biofilm with high content of persistent bacteria did not dissolve under the killing of antibiotics. The structure of the mixed biofilm was complete and dense. On the surface of the biomaterials, there were still living bacteria gathered into clumps. The mixed biofilm with low content of persistent bacteria dissolved in large area (Fig. 2C).

\section{Discussion}

Persisters in the biofilm have become a difficult point in the treatment of infection, how to remove persisters is particularly important for anti infection treatment. The low content of persisters of 
S.epidermid and C.albicans in the mixed biofilm brings many difficulties to the research. The key to solve the problem is how to induce enough persistent cells for research. The dormancy of bacteria and fungi is mainly carried out by the inhibition of protein, ATP, nucleic acid synthesis, and the ribosome assembly. If the drugs with the above mechanism are used to induce bacteria and fungi, they can be put into a dormant state to extract persister bacteria. In this study, C.albicans ATCC10231 and S.epidermidis ATCC35984 were used to culture mixed biofilm on the surface of titanium sample. The drugs that inhibit the synthesis of bacterial protein and fungal nucleic acid, such as tetracycline, gentamicin, 5-Fc, and caspofungin, were used to induce the dormancy of the mixed biofilm, and made a lot of S.epidermidis and C.albicans to enter a dormant state, which laid the foundation for studying the drug resistance mechanism and phenotypic characteristics of persistent cells.

The study showed that, tetracycline and gentamicin can make S.epidermidis enter a dormant state in large numbers, $5-\mathrm{Fc}$ and caspofunin can induce a large number of $C$.albicans to enter dormancy state. However, tetracycline and gentamicin could not induce the formation of dormant cells of C.albicans. Rifampicin, which interferes with mRNA synthesis, and CCCP, which inhibits ion transport, can only slightly increase the level of persisters of S.epidermidis in the mixed biofilm, although there is a significant difference between them and the negative control, and both of them could not induce the formation of dormant cells of C.albicans. 5-Fc inhibits DNA synthesis of fungi, while caspofungin damages the cell membrane and cell wall of fungi. Therefore, fungi can be induced to form persister cells by damaging the cell membrane and cell wall. These were different from those previously reported by Kwan et al[16], they pretreated E.coli with rifampicin, tetracycline and CCCP, and successfully induced the formation of persisters. Study[19]showed that tetracycline and rifampicin induced a viable but nonculturable state in S.epidermidis biofilm. Iron starvation and hypoxia induced persister cells of S.epidermidis in planktonic culture[20]. The number of persister bacteria obtained is more than $0.1 \%-1 \%$ reported by the study[21]. Therefore, inducing S.epidermidis and C.albicans into dormancy is not simply to inhibit its anabolism. Growth stagnation and anabolism decrease are only the results of the formation of persister cells rather than their mechanisms. Different microorganisms have different mechanisms for producing persister cells. More research is needed to clarify the mechanism by which cells enter the dormant state[13].

The research found that, after the induced persisters were re-cultured, there was no significant change in antibiotic sensitivity compared with before induction. The survival of some S.epidermidis and C.albicans after ciprofloxacin and amphotericin B treatment was not caused by antibiotic resistance due to drugresistant mutation. This indicates that the essence of persistent cells is a group of cell subgroups in a dormant state[13], which can escape the effect of antibiotics without changing their genetic characteristics. The tolerance of the bacteria to antibiotics is obtained by changing the environmental conditions and making them enter the non growth state (dormant state). Antimicrobial resistance is generated in the classical sense by genetic mutation of the corresponding antimicrobial drug target, which manifests as an increased MIC. In contrast, antimicrobial drug tolerance is not genetic, but rather an inducible phenotypic switch. The characteristics of non growth state and slow metabolism of persister bacteria make it possible to relapse again after avoiding the killing of the antibiotics, which makes the 
clinical cure more difficult. With the extension of the course of the disease, the level of persister bacteria will gradually increase, even more than 100 times[22]. Antibiotic tolerance is associated with failure of antibiotic treatment, recurrence of bacterial infection and prolonged disease[23].

Under the SEM, in the mixed biofilm inducedby 5-FC and caspofungin, some $C$.albicans cells showed bulge-like morphological changes. Benmansour W et al[24] used amphotericin B to screen C.albicans ATTCC10231 and showed similar bulge-like morphological changes on the surface of C.albicans under electron microscope. These indicate this morphological change may occur, regardless of the C.albicans being treated with dormancy-inducing drugs or directly killed by antimicrobial drugs. However, in the blank control group, only a few strains of C.albicans had this morphological change, and the proportion of persisters was far less than the experimental group. Yang et al[25] used vancomycin with the lowest bactericidal concentration to screen S.epidermidis, after 48h, the cell division phase of S.epidermidis living in the biofilm was reduced and the volume was smaller than that of ordinary bacteria, which was similar to the volume change of dormant S.epidermidis in this study, this change in volume reduction may be related to the cessation of metabolic activities and the dormancy, but the exact mechanism needs further study. CLSM observations showed that the mixed biofilms of the experimental group had clumpy structures, which mostly showed mixed growth of live and dead bacteria, these persister cells scattered in the mixed biofilm can maintain the biofilm structure, and resist the killing of antibacterial drugs, this may be an important reason why biofilm infection is difficult to cure. A study [26] showed that tRNA acetylation is one of the formation mechanisms of persisters. By using the hydrolase peptidyl-tRNA hydrolase (Pth) to hydrolyze the hetero-acetylated tRNA, the normal tRNA is released to reactivate the persisters,thus significantly reducing the level of persisters and achieving the purpose of controlling the persisters. Interfering with the dormancy process of persisters from the molecular mechanism, inhibiting the dormant state, and improving the effectiveness of antibiotics, may be a new direction for the treatment of biofilm infection. Whether there is such a mechanism in the persistesin S.epidermidisC.albicans mixed biofilm, it needs to be further studied on the basis of successful induction of a large number of persisters.

\section{Conclusions}

Induction of dormancy is a reliable method for the formation of persistent bacteria in C.albicansS.epidermidis mixed biofilm.In this study, we successfully induced S.epidermidis-C.albicans mixed biofilm to produce a lots of persistent cells, which provided a basis for further research on the phenotypic characteristics and the drug resistanceof persisters.

\section{Declarations}

\section{Ethics approval and consent to participate}

Our manuscript does not report on or involve the use of any animal or human data or tissue, the"Ethics approval and consent to participate" was not applicable in our manuscrip. 


\section{Consent for publication}

Or manuscript does not contain data from any individual person. "Consent for publication please state " was not applicable in this section.

\section{Availability of data and materials}

All data generated or analyzed during this study are presented within this manuscript. All materials used in this study including raw data shall be availed upon reasonable request.

\section{Competing interests}

Authors declare that they have no competing interests in this section.

\section{Funding}

This study was supported by National Natural Science Foundation of China (81860325and 81960335); National Key R\&D Program of China (2016YEE0103400-01);

Medical Experts Training Project of Yunnan Province ( L-2017006);

The funding body had no role in study design, data collection, analysis and interpretation of data and in writing of the manuscript.

\section{Authors' contributions}

$\mathrm{YJ}, \mathrm{BQ}, \mathrm{CR}, \mathrm{RS}, \mathrm{XC}$ and SY conducted the experiments and data analysis.

$\mathrm{YJ}, \mathrm{BQ}, \mathrm{YL}, \mathrm{RC}$ and $\mathrm{HYconceived}$ and designed the experiments.

YJ,YL,HY and WW interpreted the data and developed the manuscript arguments, reviewed and revised it.

All authors read and approved the final manuscript.

\section{Acknowledgement}

The authors are grateful to the central laboratory in the Yunnan Cancer Hospital.

\section{Author details}


Department of Thoracic Surgery, Lung Cancer Research Center,Yunnan Institute of Oncology, the Third Affiliated Hospital of Kunming Medical University, Tumor Hospital of Yunnan Province, Kunming Yunnan, 650118, P.R. China.

\section{References}

1. Wu H, Moser C, Wang HZ, Høiby N, Song ZJ. Strategies for combating bacterial biofilm infections. Int J Oral Sci. 2015;7(1):1-7.

2. Agarwal S, Sharma G, Dang S, Gupta S, Gabrani R. Antimicrobial Peptides as Anti-Infectives against Staphylococcus epidermidis. Med Princ Pract. 2016;25(4):301-8.

3. Rodríguez-Vidigal FF, Nogales-Asensio JM, Calvo-Cano A, González-Fernández R, Martínez-Carapeto A, Gómez-Sanchez I, et al. Infective endocarditis after transcatheter aortic valve implantation: Contributions of a single-centre experience on incidence and associated factors. Enferm Infecc Microbiol Clin. 2019;37(7):428-34.

4. Heilmann C, Ziebuhr W, Becker K. Are coagulase-negative staphylococci virulent? Clin Microbiol Infect. 2019;25(9):1071-80.

5. Mertens A, Ghebremedhin B. Genetic determinants and biofilm formation of clinical Staphylococcus epidermidis isolates from blood cultures and indwelling devises. Eur J Microbiol Immunol (Bp). 2013;3(2):111-9.

6. Hotterbeekx A, Xavier BB, Bielen K, Lammens C, Moons P, Schepens T,et al. The endotracheal tube microbiome associated with Pseudomonas aeruginosa or Staphylococcus epidermidis. Sci Rep. 2016;6:36507.

7. Borde JP, Sitaru G, Kopp WH, Ruhparwar A, Ehlermann P, Lasitschka F, et al. Heart transplantation as salvage therapy for progressive prosthetic valve endocarditis due to methicillin-resistant Staphylococcus epidermidis (MRSE). J Cardiothorac Surg. 2016;11(1):100.

8. Nobile CJ, Johnson AD. Candida albicans Biofilms and Human Disease. Annu Rev Microbiol. 2015;69:71-92.

9. Sardi JCO, Scorzoni L, Bernardi T, Fusco-Almeida AM, Mendes Giannini MJS. Candida species: current epidemiology, pathogenicity, biofilm formation, natural antifungal products and new therapeutic options. J Med Microbiol. 2013;62(Pt 1):10-24.

10. Harriott MM, Noverr MC. Candida albicans and Staphylococcus aureus form polymicrobial biofilms: effects on antimicrobial resistance. Antimicrob Agents Chemother. 2009;53(9):3914-22.

11. Claessens J, Roriz M, Merckx R, Baatsen P, Van Mellaert L, Van Eldere J. Inefficacy of vancomycin and teicoplanin in eradicating and killing Staphylococcus epidermidis biofilms in vitro. Int $\mathrm{J}$ Antimicrob Agents. 2015;45(4):368-75.

12. Shan Y, Brown Gandt A, Rowe SE, Deisinger JP, Conlon BP. Lewis K.ATP-Dependent Persister Formation in Escherichia coli. mBio. 2017;8(1):e02267-2216. 
13. Orman MA, Brynildsen MP. Dormancy is not necessary or sufficient for bacterial persistence. Antimicrob Agents Chemother. 2013;57(7):3230-3039.

14. Wood TK. Strategies for combating persister cell and biofilm infections. Microb Biotechnol. 2017;10(5):1054-6.

15. Shah D, Zhang Z, Khodursky A, Kaldalu N, Kurg K, Lewis K. Persisters: a distinct physiological state of E. coli. BMC Microbiol. 2006;6:53.

16. Kwan BW, Valenta JA, Benedik MJ, Wood TK. Arrested protein synthesis increases persister-like cell formation. Antimicrob Agents Chemother. 2013;57(3):1468-73.

17. Allison KR, Brynildsen MP, Collins JJ. Heterogeneous bacterial persisters and engineering approaches to eliminate them. Curr Opin Microbiol. 2011;14(5):593-8.

18. Chen Y, Wang XY, Huang YC, Zhao GQ, Lei YJ, Ye LH, et al. Study on the Structure of Candida Albicans-Staphylococcus Epidermidis Mixed Species Biofilm on Polyvinyl Chloride Biomaterial. Cell Biochem Biophys. 2015;73(2):461-8.

19. Carvalhais V, Pérez-Cabezas B, Oliveira C, Vitorino R, Vilanova M, Cerca N. Tetracycline and rifampicin induced a viable but nonculturable state in Staphylococcus epidermidis biofilms. Future Microbiol. 2018;13:27-36.

20. Fraiha RO, Pereira APR, Brito EDCA, Borges CL, Parente AFA, Perdomo RT, et al. Stress conditions in the host induce persister cells and influence biofilm formation by Staphylococcus epidermidis RP62A. Rev Soc Bras Med Trop. 2019;52:e20180001.

21. LaFleur MD, Kumamoto CA, Lewis K. Candida albicans biofilms produce antifungal-tolerant persister cells. Antimicrob Agents Chemother. 2006;50(11):3839-46.

22. Pan J, Song F, Ren D. Controlling persister cells of Pseudomonas aeruginosa PDO300 by (Z)-4bromo-5-(bromomethylene)-3-methylfuran-2(5H)-one. Bioorg Med Chem Lett. 2013;23(16):4648-51.

23. Brauner A, Fridman O, Gefen O, Balaban NQ. Distinguishing between resistance, tolerance and persistence to antibiotic treatment. Nat Rev Microbiol. 2016;14(5):320-30.

24. Benmansour W, Boucherit-Otmani Z, Boucherit K. Dormancy of Candida albicans ATCC10231 in the presence of amphotericin B. Investigation using the scanning electron microscope (SEM). J Mycol Med. 2014;24(3):e93-100.

25. Yang S, Hay ID, Cameron DR, Speir M, Cui B, Su F, et al. Antibiotic regimen based on population analysis of residing persister cells eradicates Staphylococcus epidermidis biofilms. Sci Rep. 2015;5:18578.

26. Cheverton AM, Gollan B, Przydacz M, Wong CT, Mylona A, Hare SA. et al. A Salmonella Toxin Promotes Persister Formation through Acetylation of tRNA. Mol Cell. 2016;63(1):86-96.

\section{Figures}



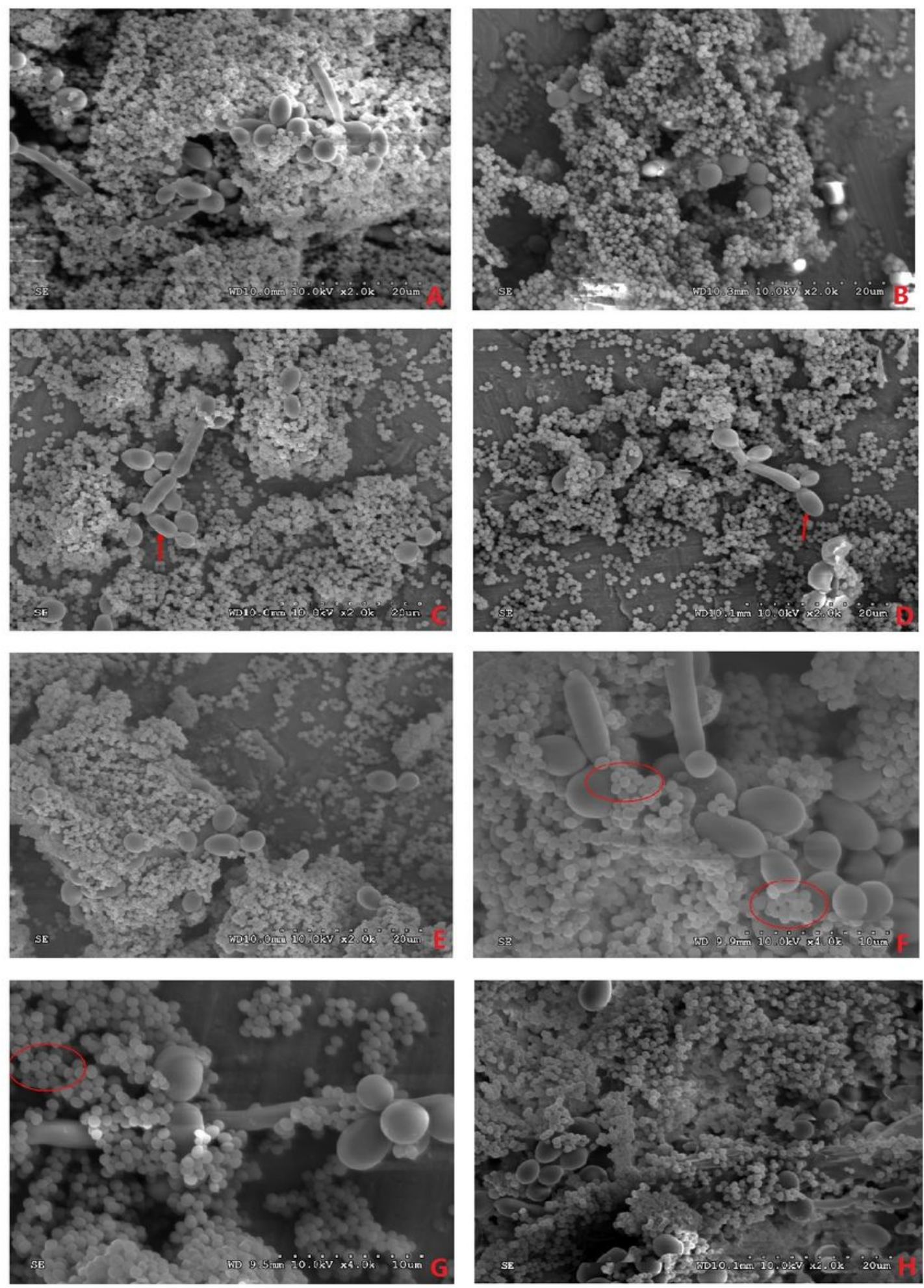

\section{Figure 1}

SEM image of the mixed biofilm(Fig.A,B,C,D,E,H×2000 times ) In the blank control group(A), the biofilms showed mixed growth of C.albicans and S.epidermidis, the hyphae and spores of C.albicans formed the reticular structure skeleton of the biofilm. In the experimental group(B), the number of biofilm, bacteria and fungi is reduced, the density of structure decreased. Induction with 5-FC(C)and Caspofungin(D) (Arrow shows morphological change of C.albicans). In the negative control group(E), the C.albicans had 
almost no convex morphological change. After inducing with tetracycline(F) and gentamicin (G)(Circle shows the S.epidermidis with reduced cell division and smaller volume than normal cells) $(\times 4000$ times).The blank control group $(\mathrm{H})$ had a large number of mitotic cells with uniform size.
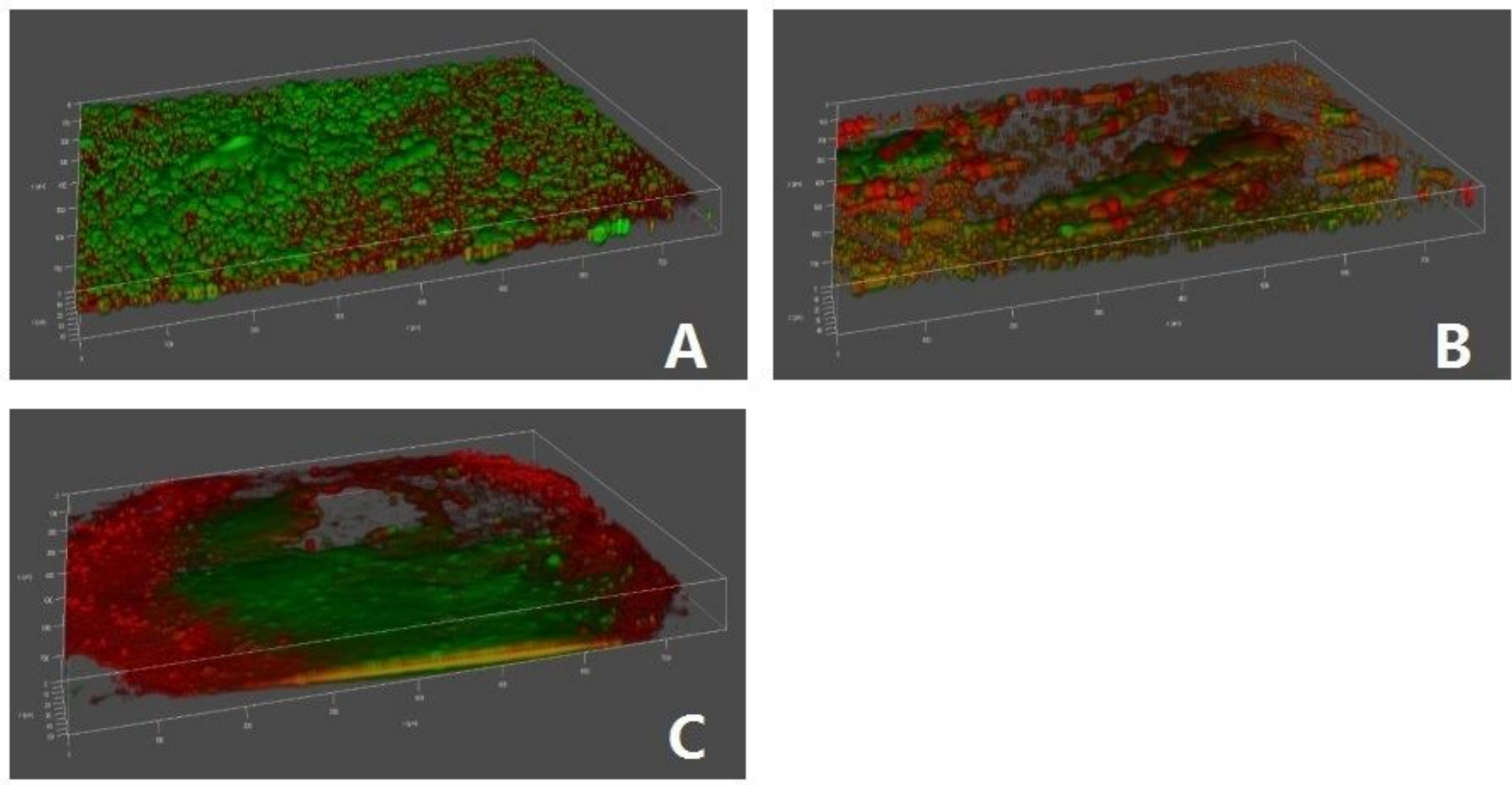

Figure 2

CLSM image of the mixed biofilm In the blank control group(A), the biofilm adhered to the surface of biomaterials, the living bacteria that emit green fluorescence are densely distributed on the surface of biofilm. In the negative control group(B), the biofilm was dissolved and destroyed, and a large number of dead bacteriae mitting red fluorescence were seen, the live bacteria were distributed on the surface of the biofilm in a dot shape. In the experimental group(C), the structure of the biofilm was partially destroyed, the biofilm with high content of persisters did not dissolve under the killing of antibacterial drugs, the biofilm with low content of persisters dissolved in large area. 